\title{
Contribuyendo al bienestar emocional de los compañeros: evaluación del Programa Compañeros Ayudantes en un instituto madrileño
}

\author{
Cristina del Barrio, Ángela Barrios, Laura Granizo, Kevin van der Meulen, \\ Soledad Andrés y Héctor Gutiérrez \\ Universidad Autónoma de Madrid (España)
}

\begin{abstract}
Un proyecto internacional desarrollado entre noviembre de 2007 y julio de 2009 se propuso implantar y evaluar un sistema de ayuda entre iguales en un centro de secundaria de Inglaterra, Italia y España, como medio de mejora de la convivencia. Los sistemas de ayuda entre iguales implican típicamente seleccionar a algunos estudiantes y entrenarlos para ayudar a sus compañeros. Mediante un diseño de grupo control con dos grupos, se formó a los ayudantes para poner en marcha un servicio de ayuda entre compañeros en los centros objeto de la intervención. Se presentan los resultados españoles de los cuestionarios de evaluación del programa, en la que participaron 248 estudiantes implicados en el programa y posibles usuarios del mismo, y 35 profesores, implicados y no implicados en el programa. Los resultados señalan que el programa es positivamente evaluado, apoyando su continuación profesores y alumnos, que coinciden en señalar las consecuencias positivas para víctimas y alumnos ayudantes, y en considerarlo un recurso para mejorar la convivencia en el centro.
\end{abstract}

Palabras clave: Ayuda entre iguales, clima escolar, mejora de convivencia, participación de adolescentes, cambio emocional.

Contributing to peers' emotional wellbeing: evaluating the Peer Support Programme in a secondary school in Madrid. Between November 2007 and June 2009 an international project aimed to establish, run and evaluate anti-bullying peer support systems in one secondary school in each of England, Italy and Spain. School anti-bullying peer support systems typically involve some pupils being selected and trained to help pupils. Using a 2 group pre-test post-test control group design, we have helped train school peer supporters and establish peer support systems in the intervention schools. For the evaluation of the programme, 248 involved and non-involved students and 35 involved and non involved teachers were surveyed using an open-end questionnaire. Here we report the outcomes of this evaluation study based on data already gathered in Spain. The results point at the positive evaluation of the programme by teachers and students, who both alike stress the positive impacts on the victims and peer supporters and see it as a resource for improving schools' daily life.

Key words: Peer support, school climate, relationships' improvement, adolescents' agency, emotional change.

Correspondencia: Cristina del Barrio. Facultad de Psicología. Universidad Autónoma de Madrid, Ciudad Universitaria de Cantoblanco. 28049 Madrid (España). E-mail: cristina.delbarrio@uam.es 
El abuso de poder o victimización entre iguales-incluyendo acoso y exclusión social-, especialmente cuando ocurren en centros escolares ha sido objeto de preocupación e investigación en la mayoría de los países (Smith et al., 1999). El interés ha venido tanto de los científicos sociales, como de las instituciones educativas y los medios de comunicación. En las dos últimas décadas se han llevando a cabo numerosos estudios en España. Destacan como referencia de la situación nacional los incluidos en los dos informes del Defensor del Pueblo que describen el problema en los centros de educación secundaria (Defensor del Pueblo-UNICEF, 2007; del Barrio et al., 2008).

En la gran mayoría de estudios se reconoce la necesidad de mejorar el clima socioemocional de los centros escolares de todos los niveles educativos reduciendo el acoso y la exclusión social que según los datos encontrados, probablemente en distinta medida se estén produciendo en todos ellos. La disminución en la incidencia de casos constatada en algunas modalidades de maltrato, al comparar los datos de ambos estudios nacionales realizados con siete años de diferencia permite hipotetizar que en parte esa disminución refleja una toma de conciencia de la gravedad del maltrato entre escolares. A pesar de esta reducción, todavía en 2007 el motivo principal de miedo al acudir al instituto son los compañeros, como señalan entre el 67 y el $84 \%$ de quienes afirman tener miedo frecuente o casi diariamente. Asimismo, cuando se es maltratado se acude principalmente a los amigos en busca de ayuda, y son ellos quienes la prestan fundamentalmente (Defensor del Pueblo-UNICEF, 2007).

En la psicología del desarrollo las relaciones entre iguales son un tema clásico al describir un desarrollo social saludable desde enfoques cognitivos, psicoanalíticos o contextuales. Para Piaget (1932) por ejemplo, las relaciones entre iguales son imprescindibles para lograr el paso de la heteronomía a la autonomía moral. El auge de las relaciones de grupo durante los últimos años de la niñez y la adolescencia temprana, y sus características cualitativamente distintas en esas etapas (Rubin, Bukowski y Parker, 2006) son un marco para entender tanto las características del maltrato entre iguales en esos años, como las estrategias de resolución del mismo. Desde hace años se acepta que el maltrato por abuso de poder en los centros escolares es un fenómeno de grupo (Salmivalli, 1999). Igualmente, ahora los modelos de intervención -paliativa o preventiva- se centran no sólo en los individuos directamente implicados, sino en el grupo en cuyo seno ocurren los abusos (Martín, Fernández, Andrés, del Barrio y Echeita, 2003; Barrios, Andrés y Granizo, 2010; para una revisión, Smith, Pepler y Rigby, 2004). El papel del grupo se manifiesta en las conductas y en otros aspectos que lo conforman: las normas y expectativas de respuesta implícitas, el clima moral del grupo y las interpretaciones cognitivas de las relaciones, subyacentes a éstas (Brugman et al., 2003; Salmivalli y Voeten, 2004; van der Meulen, Granizo y del Barrio, 2010).

Una forma de intervenir en los centros son los sistemas de ayuda entre iguales, en los que los propios estudiantes prestan una ayuda formal a sus compañeros. 
Esta herramienta implica: a) seleccionar y entrenar a alumnos para ayudar a otros en una situación emocional negativa, p.ej. las víctimas del maltrato; b) una respuesta directa ante la demanda de ayuda; c) proporcionar a los alumnos ayudantes habilidades y estrategias para encontrar una solución del problema; d) una función de apoyo y supervisión por los adultos; e) un enfoque no punitivo; f) su desarrollo fuera del curriculum formal, supeditado al compromiso de voluntarios (Sharp \& Cowie, 1998, pp. 81-82).

El entrenamiento de los ayudantes incluye habilidades básicas, p.ej. escucha activa; empatía; resolución de problemas y dar apoyo. Además, adquieren actitudes profesionales éticas: confidencialidad, compromiso, referir problemas graves a los adultos (Cowie y Wallace, 2000). Algunas estrategias utilizan conductas propias del centro, p.ej. ser tutor del trabajo académico de un compañero, liderar juegos o actividades compartidas, guiar a alumnos nuevos, etc. Otras intentan de forma organizada que los propios alumnos protagonicen la mejora de sus relaciones. Este es el caso de aquellos sistemas de ayuda en los que un número reducido de alumnos prestan apoyo psicológico a sus compañeros en situaciones de conflicto o indefensión: alumno ayudante, alumno amigo, alumno mediador (Cowie y Sharp, 1996).

Los sistemas de ayuda entre iguales representan una educación para la ciudadanía en acción, están en el espíritu de las recomendaciones de la Convención de Naciones Unidas sobre los Derechos del Niño (1991), en particular en lo tocante al derecho a la participación de niños y jóvenes, y son recomendados en informes institucionales en Reino Unido y España. Algunos datos empíricos anteriores constatan sobre todo en Inglaterra (Cowie, Naylor, Talamelli, Chauhan y Smith, 2002; Naylor y Cowie, 1999), pero también en España y otros países (Andrés 2007; Avilés, Torres y Vian, 2008; Smith et al., 2004) que estos sistemas son eficaces a la hora de proporcionar apoyo, tienen consecuencias positivas para los alumnos ayudantes (p.ej. en términos de un aumento en su autoestima), y en general, mejoran el clima socioemocional de los centros escolares, de manera distinta para las distintas partes implicadas, usuarios, ayudantes, incluso profesorado. Esta geografía emocional ha sido resaltada en estudios sobre las relaciones de maltrato entre iguales (Del Barrio, Almeida, van der Meulen, Barrios y Gutiérrez, 2003; Estévez, Murgui y Musitu, 2008) y por tanto su mejora suele ser un objetivo de los programas de intervención dirigidos a las relaciones interpersonales. Pero no hay hasta ahora estudios comparativos internacionales. Por otro lado un interés añadido en el caso español es que a diferencia del caso británico, donde es un recurso muy extendido en los centros escolares y donde se cuenta ya con un buen número de investigaciones sobre sus resultados (véase una revisión en James, Smith y Radford, 2010), y a pesar de algunas experiencias notables y bien difundidas (Fernández, Villaoslada y Funes, 2002; VVAA, 2001), este recurso educativo es aun poco conocido en la realidad educativa general de nuestro país. 
Lo que se presenta a continuación forma parte de un proyecto más amplio financiado por la Unión Europea realizado entre noviembre 2007 y julio de 2009, y cuyos objetivos fueron: a) establecer un sistema de ayuda entre iguales en un centro de educación secundaria en cada uno de los tres países participantes; b) evaluar cambios en la incidencia de situaciones de maltrato antes y después de poner en marcha el sistema; c) comparar los resultados del programa de compañeros ayudantes en los tres centros experimentales en que se realiza la intervención, y d) evaluar el impacto de dichos sistemas en los centros a través de la visión del profesorado y el alumnado acerca de su funcionamiento.

\section{METODO}

Para cumplir dichos objetivos se diseñó un estudio con grupo de control pretest-postest con dos grupos, en el que han intervenido dos escuelas de educación secundaria (una como objetivo de la intervención, otra de control) en cada uno de los tres países (Inglaterra, Italia, España). El entrenamiento de los adultos (investigadores y profesorado) que iban a formar a los alumnos ayudantes en los centros fue dirigido por Paul Naylor sobre la base de las orientaciones prácticas de Cowie y Wallace (2000). A su vez, los investigadores y profesorado seleccionaron a los alumnos y los entrenaron en cada una de las escuelas en que se iba a implantar el sistema de ayuda entre iguales

Para la evaluación del programa el procedimiento ha consistido en dos tipos de acciones: a) evaluar la prevalencia del maltrato antes y después de la implementación del sistema; y b) indagar las ideas de profesorado y alumnado del centro de intervención, acerca del éxito del sistema. En estos momentos sólo se cuenta con los datos españoles, a la espera de completar los análisis de los datos ingleses e italianos. La parte del estudio español referida al segundo aspecto, la evaluación del programa, es la que se presentará a continuación (véase del Barrio et al., 2010 para el estudio completo).

\section{Participantes}

El programa de ayuda entre iguales se ha llevado a cabo en un instituto de educación secundaria de una localidad madrileña cercana a la capital, eligiéndose como grupo control un centro similar en la misma localidad. En la evaluación del programa han participado los alumnos de las cuatro clases de primero de ESO y las cuatro clases de $3^{\circ}$ de ESO del curso del primer centro. Además, de los estudiantes que han participado directamente en el programa como compañeros ayudantes, 4 han participado en la evaluación del mismo hasta un total de 244. En cuanto al profesorado, han sido 35 profesores quienes han participado en la evaluación, de los cuales cuatro son profesores implicados en el programa (Tabla 1). 
Tabla 1. Distribución de participantes en la evaluación del programa de ayuda entre iguales

\begin{tabular}{|c|c|}
\hline Estudiantes & Profesores \\
\hline $\begin{array}{c}244 \text { no implicados } \\
\text { - } 20,5 \% \text { inmigrantes })\end{array}$ & $\begin{array}{l}\text { - } 31 \text { no implicados: } \\
22 \text { mujeres } 9 \text { hombres }\end{array}$ \\
\hline \multicolumn{2}{|l|}{$1^{\circ} \mathrm{ESO}(\mathrm{n}=129)$ Media de edad: $13 ; 5$} \\
\hline $3^{\circ}$ ESO $(n=115)$ Media de edad: $15 ; 1$ & entre 1 y 36 años de experiencia docente \\
\hline \multirow[t]{2}{*}{$\begin{array}{l}\text { - } \quad 4 \text { ayudantes (chicas) } \\
\text { rango de edad: } 15 ; 8>18 ; 3\end{array}$} & $\begin{array}{l}\text { - } 4 \text { implicados: } \\
2 \text { profesores (supervisor del programa) }\end{array}$ \\
\hline & 2 profesoras \\
\hline
\end{tabular}

\section{Instrumentos}

A partir de cuestionarios y entrevistas utilizados en estudios anteriores centrados en la evaluación de sistemas de ayuda entre iguales (Cowie et al., 2002; Naylor y Cowie, 1999), se elaboraron cuatro cuestionarios, cada uno dirigido a cada conjunto de participantes: estudiantes implicados en el programa como ayudantes; estudiantes usuarios potenciales del programa; profesorado implicado en el programa (supervisor y los tres colaboradores) y profesorado del centro en el que se ha implantado el programa. En ellos, además de datos demográficos de los participantes, se indaga sobre su conocimiento del programa, su percepción de posibles beneficios en el caso de los estudiantes usuarios del mismo, de los estudiantes ayudantes, del profesorado y de la escuela; sus ideas acerca de las cualidades esperables en un alumno ayudante y acerca de la continuidad del programa y los problemas encontrados en el mismo. Las preguntas son abiertas en todos los casos.

\section{Procedimiento}

Al final del curso en el que se implantó el programa compañeros ayudantes (junio 09), y una semana después de administrar el cuestionario de incidencia para determinar posibles cambios con respecto al cumplimentado un año atrás (junio 08) se administró de modo colectivo el cuestionario de evaluación del programa en cada uno de los grupos de primero y tercero de ESO. Simultáneamente, se repartió al profesorado en la sala de profesores animando a que participaran, y 31 fueron devueltos cumplimentados. En la última reunión de supervisión del programa, los profesores implicados en el programa cumplimentaron su cuestionario, y debido a la dificultad de las fechas (acabados los exámenes finales), sólo asistieron cuatro alumnos ayudantes que rellenaron su cuestionario. Se procedió a un análisis cualitativo de las respuestas obtenidas y en su caso a un análisis estadístico para establecer posibles diferencias significativas entre los grupos de $1^{\circ}$ y $3^{\circ}$ de ESO y de profesores. 


\section{RESULTADOS}

Se ofrecen aquí los resultados relativos a la evaluación realizada por los estudiantes y profesores no implicados en el programa, organizados según los aspectos indagados en los cuestionarios respectivos.

\section{Conocimiento del programa}

Con respecto a cómo se ha llegado a conocer el programa de compañeros ayudantes por parte de los estudiantes y profesores que han participado en la evaluación del mismo, se encuentra una diferencia estadísticamente significativa en el número de personas que desconocen el programa en los tres grupos de edad, a menos edad más desconocimiento del mismo. Así, en el caso de los estudiantes más jóvenes, los de $1^{\circ}$ de ESO, más de la mitad de ellos (52.9\%), desconoce el programa. En $3^{\circ}$ son un $17.7 \%$ quienes no lo conocen, y entre el profesorado, un 3.2.

La fuente de información de quienes sí lo conocen también parece variar entre los estudiantes de los dos grupos, y lo que era más esperable entre ellos y el profesorado (Tabla 2). En $1^{\circ}$, en quienes sí lo conocen, la fuente principal de información acerca del programa ha sido su tutor (19.5\%), y en segundo lugar los compañeros (15.3\%). En el caso de los estudiantes de $3^{\circ}$, son de nuevo el tutor y en igual (elevado) porcentaje, la propia gente del programa $(43.4 \%)$ que se distancia significativamente del porcentaje de alumnos de $1^{\circ}$ que citaban esta misma fuente. Entre el profesorado, el programa se ha conocido sobre todo por la vía institucional $(74 \%)$ ya que se citan tanto el consejo escolar como las reuniones (informativa específica, claustro o de departamento). Los medios de difusión del programa que han elaborado los estudiantes implicados, i.e. carteles informativos y la página web, son una fuente importante para el profesorado y se citan más por los alumnos mayores que por los pequeños siendo estadísticamente relevante esta distribución dispar. Los profesores citan como fuente a los compañeros profesores y a los estudiantes en proporción similar, cercana al 23\%. Los propios estudiantes son un canal para el $20 \%$ de $3^{\circ}$ y algo menos de $1^{\circ}$. En menor medida citan al orientador los chicos de $3^{\circ}$ y el profesorado.

\section{Beneficios del programa para usuarios y para el centro escolar}

Con respecto a los beneficios para los usuarios del servicio de compañeros ayudantes (Tabla 3), casi tres cuartas partes de los estudiantes que han evaluado el programa los describen en forma de la ayuda que éste proporciona a quienes acuden al servicio. Los más jóvenes -estudiantes de $1^{\circ}$ de ESO- expresan esta ayuda de forma más inespecífica que sus compañeros de $3^{\circ}$. Sin embargo, más de un tercio de cada grupo de edad también la concretan en resolución de problemas, como en el caso de esta chica de $3^{\circ}$ (15;4): “Ayuda a superar los problemas". 
Algo más de un cuarto del conjunto de estudiantes participantes en la evaluación se refiere al alivio emocional como una consecuencia positiva del programa, señalando ligeramente más este beneficio los alumnos de $3^{\circ}$, como esta alumna $(14 ; 6)$ : "Te puedes desahogar y te quedas mucho mejor". En menor medida, hablan de la mejora del clima escolar, desde una doble perspectiva: "Hay menos conflictos" (chica de $1^{\circ}$ ); "Es una iniciativa muy buena para llevarse mejor" (chico de $1^{\circ}$ ). La disminución de conflictos es significativamente más mencionada por los alumnos de $1^{\circ}$. Aspectos del propio programa (su anonimato), destacando la participación de los propios compañeros -una característica del mismo- son vistos también como beneficios, como señala una estudiante de $3^{\circ}$ : "Les pueden ayudar sin que intervengan los adultos. Son los mayores quienes más se refieren a la naturaleza del programa como un beneficio para los usuarios del mismo, sin que esta diferencia alcance valores significativos.

Tabla 2. Fuente/s de Conocimiento del programa (\%) Comparación estudiantes $(N=232)$ y profesores no implicados $(N=31)$

\begin{tabular}{lrrr}
\hline & \multicolumn{2}{c}{ Estudiantes } & Profesores \\
\cline { 2 - 4 } & \multicolumn{1}{c}{$1^{\circ}$} & $3^{\circ}$ & 22.6 \\
\hline Compañero o alumno & 15.3 & 19.5 & $*$ \\
Carta dirigida a los padres & 1.7 & 7.1 & $* * *$ \\
Tutor & 19.5 & 43.4 & $22.6^{* *}$ \\
Profesor & 4.3 & 11.5 & $12.9^{* *}$ \\
Orientador & 0.9 & 11.5 & 74.2 \\
Institución (Consejo, Reunión) & - & - & $29^{* * *}$ \\
Carteles, pág.web & 3.4 & 27.4 & $6.5^{* * *}$ \\
Gente del programa & 8.5 & 43.4 & 3.2 \\
Otros & 2.6 & 0.9 & $3.2 * * *$ \\
No lo conozco & 52.9 & 17.7 & 31 \\
\hline
\end{tabular}

$* p \leq .05 \quad * * p \leq .01 \quad * * * p \leq .001$ Nota: El número total tiene en cuenta los valores perdidos, i.e. casos que no han contestado a la pregunta del cuestionario, razón por la cual puede variar en cada tabla.

Tabla 3. Beneficios para los usuarios según los estudiantes no implicados

\begin{tabular}{|c|c|c|c|}
\hline \multirow{2}{*}{ BENEFICIOS } & \multicolumn{3}{|c|}{ ESTUDIANTES $(N=166)$} \\
\hline & $1^{\circ}(\%)$ & $3^{\circ}(\%)$ & TOTAL $(\%)$ \\
\hline Ayuda & 76.3 & 66.3 & 71.1 \\
\hline Ayuda & 42.1 & 32.6 & 37 \\
\hline Resolución de problemas & 38.2 & 36 & 37 \\
\hline Alivio emocional & 20 & 32.6 & 26.5 \\
\hline Naturaleza del programa & 5 & 8.1 & 6.6 \\
\hline Iguales como agentes & 2.5 & 4.7 & 3.6 \\
\hline Mejora del clima escolar & 10.7 & 4.7 & 7.5 \\
\hline Disminución de problemas & 8 & $1.2 *$ & 4.3 \\
\hline Influencia positiva & 4 & 3.5 & 3.7 \\
\hline Otros & 1.3 & 3.5 & 2.4 \\
\hline
\end{tabular}

En cuanto a los beneficios que la puesta en marcha del programa supone para la escuela (Tabla 4), la mayoría de los estudiantes $(44.5 \%)$, ya sean de primer o tercer 
curso, se refiere a la mejora del clima escolar. Se encuentra incluido en esta categoría un buen número de respuestas $(28.2 \%)$ que alude a la disminución de problemas como causa de esta mejora. En segundo lugar, se menciona la ayuda que el programa proporciona al centro (25.2\%). Sólo algunos estudiantes de $1^{\circ}(10 \%)$, lo cual da lugar a una diferencia significativa, puntualizan acerca de esta ayuda incorporando la idea de buen funcionamiento o eficacia del programa al conseguir resolver ciertos problemas que surgen en la escuela.

Teniendo en cuenta las dos categorías comentadas, se observa que los estudiantes consideran el programa beneficioso tanto para prevenir (disminuye la aparición de conflictos) como para intervenir (contribuye a resolverlos). Llama la atención que casi un $13 \%$ de estudiantes se refieren a una mejora emocional de los alumnos como beneficio personal que, sin embargo se ve como un beneficio para toda la comunidad escolar. En este sentido se explica un alumno de $1^{\circ}(13 ; 6)$ : "Los alumnos tendrán menos miedo y estarán más tranquilos".

Aunque en menor medida $(8.4 \%)$, también se encuentran respuestas que señalan como beneficio del programa, el aumento de prestigio de la escuela. Como los alumnos comentan: "Da mejor imagen" (chica de $1^{\circ}(13 ; 9)$ ó "Acuden más personas al centro" (chica de $3^{\circ}(15 ; 6)$. En este caso, estas respuestas son más frecuentes en los estudiantes mayores que en los menores, aunque la diferencia no resulta significativa.

\begin{tabular}{|c|c|c|c|}
\hline \multirow{2}{*}{ BENEFICIOS } & \multicolumn{3}{|c|}{ ESTUDIANTES $(N=119)$} \\
\hline & \multirow{2}{*}{$\begin{array}{c}1^{\circ}(\%) \\
32.7\end{array}$} & \multirow{2}{*}{$\begin{array}{c}3^{\circ}(\%) \\
19.4\end{array}$} & \multirow{2}{*}{$\begin{array}{c}\text { TOTAL }(\%) \\
25.2\end{array}$} \\
\hline & & & \\
\hline Ayuda (tautología) & 24 & 19.4 & 21.4 \\
\hline Resolución de problemas & 10 & $-* *$ & 4.3 \\
\hline Alivio emocional & 11.5 & 13.4 & 12.6 \\
\hline Características del programa & - & 6 & 3.4 \\
\hline Participación de los iguales & 1.9 & 1.5 & 1.7 \\
\hline Mejora del clima escolar & 40.4 & 47.8 & 44.5 \\
\hline Disminución de problemas & 30 & 26.9 & 28.2 \\
\hline Influencia positiva & 8 & 7.5 & 7.7 \\
\hline Prestigio de la escuela & 5.8 & 10.4 & 8.4 \\
\hline Otros & 3.8 & 4.5 & 4.2 \\
\hline
\end{tabular}

\section{Percepción del programa por parte del profesorado}

En cuanto a las cualidades que más se valoran en un alumno ayudante los docentes destacan, por orden de frecuencia, la empatía $(35.5 \%)$, la escucha activa (32.2\%), la accesibilidad (32\%) y el compromiso o sentido profesional (29\%), que incluye el $13 \%$ que menciona responsabilidad, y la comunicación o capacidad de diálogo (29\%). Todas ellas son habilidades que se entrenan en la formación como compañeros 
ayudantes. La popularidad es señalada en menor medida, por menos de la cuarta parte del profesorado.

\section{Beneficios del programa de compañeros ayudantes según el profesorado}

Como se observa en la tabla 5, el profesorado no participante en el estudio discrimina el tipo de beneficios según el destinatario a quien se los atribuya. Incluso una categoría como es la mejora emocional, adopta formas diferentes según quien la experimente: los usuarios del programa, los ayudantes o los profesores.

Para los usuarios el beneficio que más docentes señalan es el del alivio emocional (58\%), como señala una profesora con 30 años de experiencia: "Sentirse comprendidos y protegidos". Pero también es un beneficio la ayuda en la solución de sus problemas (39\%) como se señala en estos dos casos: "Tienen una salida a su situación" (profesor, 31 de experiencia), "Que pueden encontrar solución ante un problema que les impide su desarrollo adecuado" (profesora, 8 de experiencia).

Tabla 5. Beneficios del programa de Alumnos Ayudantes según los profesores no implicados $(N=31)$

\begin{tabular}{|c|c|c|c|c|c|}
\hline Beneficios para: & Usuarios (\%) & Ayudantes (\%) & Profesores (\%) & Escuela $(\%)$ & TOTAL (\%) \\
\hline \multirow{2}{*}{ Mejora emocional: } & Alivio & Sentirse útil & Sentirse útil & & \\
\hline & 58 & 35 & 3 & - & 97 \\
\hline Ayuda/Resolución de problemas & 39 & - & 23 & 10 & 71 \\
\hline Recurso de apoyo & - & - & 35 & 6 & 42 \\
\hline Mejora de competencias & - & 58 & 3 & - & 61 \\
\hline Madurez & - & 19 & - & - & 19 \\
\hline Mejora de la convivencia & - & - & 23 & 58 & 81 \\
\hline Agencia de iguales & 13 & 10 & 19 & 3 & 45 \\
\hline Implicación académica & - & - & 10 & 6 & 16 \\
\hline Otros & 10 & 3 & 6 & 6 & 26 \\
\hline
\end{tabular}

En el caso de los ayudantes, los profesores resaltan la mejora de competencias ("Desarrollar capacidades y actitudes solidarias, sociales", como señala una profesora, con 15 años de experiencia), y la mejora emocional: "Ayudar aumenta la autoconfianza, siempre es gratificante” (profesor, 19 de experiencia). En el caso de los propios profesores, el beneficio más citado es el contar con un nuevo recurso en su apoyo, como señala este mismo profesor: "Menos trabajo, pues se interviene en temas que a ellos (los profesores) les resultan especialmente complejos”. Para el centro la consecuencia más positiva es la mejora de la convivencia. Esta, junto con la mejora emocional son las categorías que agrupan más respuestas de los docentes. La participación de los estudiantes como agentes de resolución de problemas de sus iguales es un beneficio señalado en el $45 \%$ de las respuestas y destacado en los siguientes ejemplos de profesoras con 28 y 25 años de experiencia respectivamente: "Proximidad para comunicar su situación, puede aumentar la inmediatez en prestar la ayuda. Facilita la comunicación por ser de su grupo”; "Una ayuda básica, pues muchas veces las víctimas 
no se atreven a romper la barrera con los adultos". Otras respuestas destacan la buena imagen que el programa da al centro junto con sus implicaciones académicas: "Se beneficia de un buen ambiente de trabajo y que los padres vean que hay una ayuda, no sólo académica, para sus hijos" (profesora, 8 años de experiencia). Los estudiantes también hablaban de este prestigio del centro como consecuencia del programa.

\section{Continuidad del programa}

La mayoría de los estudiantes, ya sean de $1^{\circ}(82 \%)$ o de $3^{\circ}(78 \%)$, así como la mayoría de los docentes $(87 \%)$, cree que el programa debe continuar. Cabe destacar que ningún profesor o profesora de la muestra responde de forma negativa a esta pregunta. En cuanto al alumnado, más estudiantes de $1^{\circ}(16 \%)$ que de $3^{\circ}(6 \%)$ opinan que el programa no continúe. Va aumentando con la edad de modo significativo $(p \leq .001)$ quienes no contestan o señalan no saber qué responder.

Las principales razones que estudiantes y docentes mencionan para apoyar la continuidad del programa, tienen que ver con la ayuda que éste supone (Tabla 6). Esta respuesta es más frecuente entre los alumnos que entre el profesorado, diferencia que resulta significativa. De nuevo, en esta categoría, entre las respuestas que aluden a la ayuda que el programa ofrece, las que hablan de una ayuda resolutiva son menos frecuentes en los estudiantes que en el profesorado, quienes sólo expresan la ayuda en estos términos $(29.6 \%)$.

Tabla 6. Por qué debe continuar el programa. Comparación de estudiantes y profesores no implicados

\begin{tabular}{|c|c|c|c|c|}
\hline & & \multicolumn{2}{|c|}{$\begin{array}{c}\text { ESTUDIANTES } \\
N=186\end{array}$} & \multirow{2}{*}{$\begin{array}{c}\text { PROFESORES } \\
N=27 \\
(\%)\end{array}$} \\
\hline & & $\mathbf{1}^{\circ}(\%)$ & $\mathbf{3}^{\circ}(\%)$ & \\
\hline \multirow[t]{3}{*}{ Ayuda } & & 73.3 & 67.1 & $29.6 * * *$ \\
\hline & Ayuda & 53.5 & 48.2 & $-* * *$ \\
\hline & Resolución de Problemas & 19.8 & 18.8 & 29.6 \\
\hline Alivio emocional & & 5 & 9.4 & - \\
\hline \multirow[t]{2}{*}{ Naturaleza del programa } & & 2 & 1.2 & $11.1 *$ \\
\hline & Iguales como agentes & 2 & - & $11.1 * *$ \\
\hline Mejora de la Convivencia & & 5 & 5.9 & 14.8 \\
\hline Lograr Experiencia & & - & - & 25.9 \\
\hline Valoración positiva & & - & - & 22.2 \\
\hline Respuesta egocéntrica & & 6.9 & 9.4 & - \\
\hline Desconocimiento & & 13.9 & 7.1 & - \\
\hline Escepticismo & & 1 & 2.4 & - \\
\hline Otras & & 2 & 4.8 & - \\
\hline
\end{tabular}

Para los docentes otra razón importante para continuar con el programa (25.9\%) se encuentra en la necesidad de tiempo y experiencia para apreciar sus resultados. Por ejemplo, una profesora con 5 años de experiencia docente, comenta: "Este año sólo es el inicio, y en los años venideros seguro que se refuerza su 
importancia". Un colega con 31 años de experiencia también apunta: "Será más útil cuando tenga continuidad".

Se encuentran otras diferencias significativas vinculadas a las respuestas que aluden a la naturaleza del programa para justificar su continuidad. En este sentido, son más los docentes $(11.1 \%)$ que los alumnos $(2 \%)$ quienes hablan de la participación de los iguales. Por último, tanto algunos estudiantes de $1^{\circ}(13.9 \%)$ como de $3^{\circ}(7.1 \%)$ reconocen no saber por qué debería continuar el programa. Se encuentran respuestas egocéntricas, centradas en sus propios intereses $\left(6.9 \%\right.$ y $9.4 \%$ en $1^{\circ}$ y $\left.3^{\circ}\right)$, p.ej.: " $Q u e$ continúe por si me pasa, aunque todavía no me ha pasado" (chico, $1^{\circ}$ ). No obstante, y más en el grupo de los alumnos mayores, se contemplan razones que se refieren a la mejora emocional que el programa proporciona a sus iguales.

\section{CONCLUSIONES}

El programa es evaluado muy positivamente por los estudiantes y el profesorado del centro en el que se desarrolla. Los estudiantes más jóvenes tienen menor conocimiento del mismo, lo que llevaría a reforzar los canales de información en este ciclo. Por el contrario, una gran mayoría de los alumnos de $3^{\circ}$ y del profesorado sí lo conoce, aunque por distintas vías: para los estudiantes de ambos cursos, su tutor junto con la gente del programa (investigadores, profesores implicados y compañeros ayudantes), especialmente para los de $3^{\circ}$ de ESO; para el profesorado, la vía institucional, en reuniones, consejos, etc.

Tanto los estudiantes como el profesorado mencionan los beneficios que tiene el programa, discriminando los diversos destinatarios: usuarios, compañeros ayudantes, profesorado y centro. Destacan el aumento de emociones positivas que se traduce en alivio para los usuarios del programa y en autoestima para los ayudantes, y la mejora de la convivencia. Es destacable que entre los beneficios para el centro los estudiantes se refieren también al alivio emocional de los chicos que acuden al servicio, lo que supone una mejora en la vía del ajuste y cambio emocional de quienes se encuentran en distintas situaciones, ya sea como víctimas, perpetradores u observadores de situaciones negativas, cambio que puede preludiar el de la solución de dichas situaciones negativas (Del Barrio et al., 2003; Estévez et al., 2008). Y que contemplen tanto el servicio con una función reparadora como preventiva. Las cualidades que valoran los profesores en los estudiantes ayudantes son la empatía, disponibilidad, el saber escuchar, dialogar y comprometerse, todas ellas habilidades prosociales que forman parte del entrenamiento de los compañeros ayudantes.

La gran mayoría de estudiantes y profesores considera que el programa debe continuar por sus beneficios para los usuarios y para el centro, considerándolo un recurso que mejora la imagen de seguridad del mismo. Hay que destacar que tanto los 
estudiantes como los profesores al hablar de la continuidad y de los beneficios del programa señalan la participación de los propios estudiantes en la resolución de los problemas emocionales y los conflictos como una ventaja. Estos datos coinciden con otros estudios que han evaluado el funcionamiento de sistemas de ayuda entre iguales. Junto con la ligera disminución de la incidencia de maltrato observada en el centro de intervención, son la base para avanzar en el programa y aumentar los centros que dispongan de esta herramienta que da a los estudiantes un papel activo en la mejora de las relaciones interpersonales de los centros en los que no sólo estudian sino también aprenden a vivir.

\section{Agradecimientos}

Este estudio ha sido parcialmente financiado por la Comunidad Europea, Programa Grundtvig. Los autores reconocen la inspiración de Helen Cowie (University of Surrey) y especialmente el empuje de Paul Naylor (Aston University) para llevar a cabo este proyecto. De igual modo, agradecen la colaboración y los muchos aprendizajes logrados junto con los estudiantes y profesorado del IES Rosa Chacel (Colmenar Viejo, Madrid) que participan de modo entusiasta en el estudio.

\section{REFERENCIAS}

Andrés, S. (2007). Los sistemas de ayuda entre iguales como instrumentos de mejora de la convivencia en la escuela: evaluación de una intervención. Universidad Autónoma de Madrid, Facultad de Psicología: Tesis doctoral no publicada.

Andrés, S. y Barrios, A. (2006). El modelo del alumno ayudante a discusión: la opinión de los alumnos participantes y sus beneficiarios. Revista de Investigación Psicoeducativa, 4, $160-174$.

Avilés, J.M., Torres, N. y Vian, M.V. (2008). Equipos de ayuda, maltrato entre Iguales y convivencia escolar. Revista Electrónica de Investigación Psicoeducativa, 16, 863-886.

Barrios, A., Andrés, S. y Granizo, L. (2010). El asesoramiento para la mejora de la Convivencia el Clima de centro. En E. Martín y J. Onrubia (Coords.). Orientación Educativa: Procesos de Innovación y mejora de las enseñanzas. Vol. 3. Barcelona: Graó, en prensa.

Brugman, D., Podolskij, A.I., Heymans, P.G., Boom, J., Karabanova, O. y Idobaeva, O. (2003). Perception of moral atmosphere in school and norm transgressive behaviour in adolescence: An intervention study. International Journal of Behavioral Development, 27, 289-300.

Cowie, H., Naylor, P., Talamelli, L., Chauhan, P. y Smith, P.K. (2002). Knowledge, use of and attitudes towards peer support: a 2-year follow-up to the Prince's Trust survey. Journal of Adolescence, 25, 453-467.

Cowie, H. y Sharp, S. (1996). Peer Counselling in schools. Londres: David Fulton Publishers.

Cowie, H. y Wallace, P. (2000). Peer Support in Action. Londres: Sage Publications.

Defensor del Pueblo-UNICEF (2007). Violencia escolar: el maltrato entre iguales en la educación secundaria obligatoria 1999-2006. Nuevo estudio y actualización del Informe 2000. Madrid: Publicaciones de la Oficina del Defensor del Pueblo. 
Del Barrio, C., Almeida, A., van der Meulen, K., Barrios, A. y Gutiérrez, H. (2003). Representaciones acerca del maltrato entre iguales, atribuciones emocionales y percepción de estrategias de cambio a partir de un instrumento narrativo: SCANBullying. Infancia y Aprendizaje, 26, 63-78.

Del Barrio, C., Granizo, L., Gutiérrez, H., van der Meulen, K., Barrios, A. y Andrés, S. (2010). Adolescents as agents in improving daily life in schools: a case study in a Spanish school. En preparación.

Del Barrio, C., Martín, E., Montero, I., Gutiérrez, H., Barrios, A. y de Dios, M.J. (2008). Bullying and social exclusion in Spanish secondary schools: National trends from 1999 to 2006. International Journal of Clinical and Health Psychology, 8(3), 657-677.

Estévez, E., Murgui, S. y Musitu, G. (2008). Ajuste psicosocial en agresores, víctimas puras y víctimas agresivas en la escuela. European Journal of Education and Psychology, 1(2), 19-39.

Fernández, I., Villaoslada, E. y Funes, S. (2002). Conflicto en el centro escolar: el modelo de alumno ayudante como estrategia de intervención educativa. Madrid: La Catarata.

James, A., Smith, P.K. y Radford, L. (2010). What impact does peer support have on school climate? Findings from a four month case study in an English secondary school. Ponencia presentada en el II Congreso Internacional de Convivencia Escolar. Almería, 16-19 marzo, 2010.

Martín, E., Fernández, I., Andrés, S., del Barrio, C y Echeita, G. (2003). La intervención en los centros escolares: mejora de la convivencia y prevención de conflictos. Infancia y Aprendizaje, 26, 79-95.

Naylor, P. y Cowie, H. (1999). The effectiveness of peer support systems in challenging school bullying: the perspectives and experiences of teachers and pupils. Journal of Adolescence, 22, 467-479.

Ortega, R. (1998). La convivencia escolar: qué es y cómo abordarla. Junta de Andalucía, Consejería de Educación y Ciencia.

Piaget, J. (1932). El criterio moral en el niño. Barcelona: Martínez Roca, 1984.

Rubin, K.H., Bukowski, W. y Parker, J. (2006). Peer interactions, relationships, and groups. En N. Eisenberg (Ed), Handbook of Child Psychology (6 $6^{\text {th }}$ edition): Social, emotional, and personality development. (pp. 571-645) Nueva York: Wiley.

Salmivalli, C. (1999). Participant role approach to school bullying: Implications and intervention. Journal of Adolescence, 22, 453-459.

Salmivalli, C. y Voeten, M. (2004). Connections between attitudes, group norms, and behaviour in bullying situations. International Journal of Behavioral Development, 28, 246-258

Sharp, S. y Cowie, H. (1998). Counselling and Supporting Children in Distress. Londres: Sage.

Smith, P.K., Morita, Y., Junger-Tas, J., Olweus, D., Catalano, R. y Slee, P. (1999) The Nature of School Bullying. A cross-national perspective. Londres: Routledge.

Smith, P.K., Pepler, D. y Rigby, K. (2004). Bullying in schools: How successful can interventions be? Cambridge: Cambridge University Press.

Van der Meulen, K., Granizo, L. y del Barrio, C. (2010) EQUIPAR para Educadores: Adolescentes en situación de conflicto. Adaptación española de A-M DiBiase, J. Gibbs y B. Potter (2005): EQUIP for Educators: Teaching youth (grades5-8) to think and act responsibly. Madrid: La Catarata.

VVAA (2001). Monográfico Convivencia escolar. Cuadernos de Pedagogía, 304, 9-105.

Recibido: 1 de marzo de 2010

Recepción Modificaciones: 19 de noviembre de 2010

Aceptado: 22 de noviembre de 2010 